\title{
Spectroscopy of single organic dye-molecules and semiconductor quantum dots: basic aspects and applications in nanoscopy
}

\author{
A.V. Naumov* \\ Institute for Spectroscopy RAS, 108840 Moscow, Troitsk, Russia \\ Moscow State Pedagogical University, 119991 Moscow, Russia
}

\begin{abstract}
.
Spectroscopy and microscopy of single quantum emitters is a research direction that has become by now one of the most topical issues in physics, physical chemistry, biophysics and related sciences [1-4]. This direction was marked by particularly intensive development when some new artificial chromophores became available. Those were molecular complexes, organometallic and hybrid light emitters, dielectric nanoparticles and colloidal semiconductor nanocrystals (quantum dots, QDs). Besides, the man-created nano-objects could have the specified photophysical characteristics.

Registration of fluorescence images of single point emitters in the scheme using a scanning confocal or widefield microscope can give the source coordinates with a subdiffraction (nanometer) accuracy. [5] The reconstruction of the emitters' coordinates is performed from the analysis of the point spread function (PSF). PSF can be modified to implement the super-resolution of all three coordinates using the adaptive optics elements, e.g., the double helix scheme [6]. In this case, the point source image becomes a complicated double-lobe shape and requires a specific analysis.

Restoration of coordinates for a statistically large number of single probe chromophores allows one to reconstruct the structure of the material under study with nanometer spatial resolution. This principle is a basis for rapidly growing multidisciplinary area known as the far-field optical microscopy of ultrahigh resolution (nanoscopy) with the reconstruction of fluorescence images of single point emitters. [2]

Especially informative and the most sensitive to structural and dynamic parameters of the nearby surrounding are the zero-phonon spectral lines (ZPL) of single impurity organic molecules (SM). [7] ZPLs are extremely sensitive to static and dynamic local fields as well as guest-host interactions. [8-12] The principal task in the development of this direction is to find the regularities connecting microscopic information on the level of individual emitters and their nano-surrounding with the macroscopic characteristics of the bulk samples. [3] The way to solve this problem consists in the statistical analysis of data for a large number of SMs. [4]
\end{abstract}

\footnotetext{
*Corresponding author: naumov@isan.troitsk.ru; web-page: www.single-molecule.ru
} 
Our team is involved in development of the hardware and software solutions and methods for rapid detection and computer analysis of fluorescence images, spectra and spectral trajectories for the gigantic number of single emitters. The developed techniques allow us to perform qualitatively new type of measurements in the field of spectroscopy of the impurity center which is registration of individual spectra and the spatial coordinates of effectively emitting centers in the macroscopic volume of the sample doped with fluorescent probe molecules $[3,4,13,14]$. In particular, we have been carried out experiments on the far-field visualizing of local structural features in solid films (defects, cracks) with characteristic dimensions of a few nanometers.

The method is based on a series-parallel detection of fluorescence images and spectra of a giant amounts of single fluorescent molecules and statistical analysis of the data. SM emission is excited selectively by a narrowband laser frequency tuning to the resonance frequencies of SM electronic transitions. Analysis of SM spatial placement makes it possible to visualize the features of the solid structure of the sample with nanometer accuracy, exceeding the diffraction limit.

The features of nanoscopy expand qualitatively the detection of spatial and spectral (photophysical) characteristics of probe emitters. In fact, in these experiments, microscopy acquires extra "spectral coordinates", which are individual spectral characteristics of nanoprobes, which makes it possible to carry put a kind of "spectral nanotomography", revealing different characteristics of the local structure and dynamics of the sample (Fig. 1).

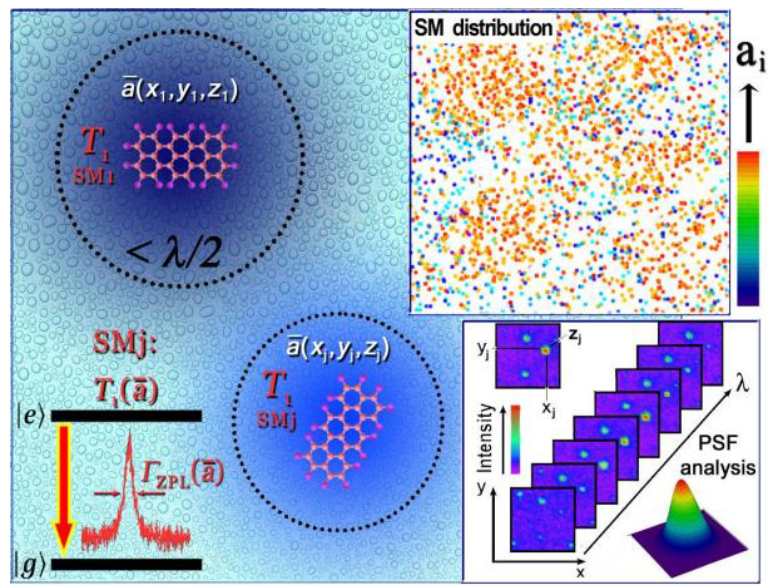

Fig. 1. The sketch to the idea of "multicolor" far-field ZPL-based fluorescence nanoscopy.

This talk discusses the main techniques for detection of single point emitters (chromophore organic molecules, semiconductor quantum dots), temporal and spectral characteristics of SMs and QDs in different environments, the blinking phenomenon, quenching and enhancement of the luminescence of single emitters. There is a discussion of the nanoscopy methods based on the reconstruction of coordinates of single probe emitters, including - 3D-microscopy techniques with the instrumental PSF modification. It is shown how the unique properties of the ZPL can become the basis for a new technique of "multicolor" far-field optical nanodiagnostics of solid media and nanostructures, where the reconstruction of the image is carried out by registering the of the zero-phonon fluorescence images and by restoring the coordinates of a large number of probe SMs with subdiffraction accuracy. In particular, it is proposed to use our approach to nanodiagnostics (mapping) of the local fields in solids, as well as creating a microrefractometry technique [15]. The basis of this approach is the fact that at ultralow temperatures the width of the SM ZPL is determined only by the lifetime of the excited state $T_{1}$, which, due to the local field effects, is associated with the effective value of the refractive index of the medium $[16,17]$. Thus, 
the ZPL width value may be converted to an effective value of the refractive index in the local neighborhood of each registered SM.

At room temperature, the idea of "multicolor" nanoscopy can be implemented by methods spectromiroscopy of single colloidal quantum dots. In this case, the coordinates a QD are restored with nanometer accuracy [18], and the additional options for hyperspectral diagnosis can be obtained by analyzing the kinetics and statistics of blinking luminescence of single QDs [19-26]. In particular the idea of microrefractometry with single quantum emitter can be realized with time-resolved spectroscopy of single QD. [27]

Thus, this paper shows that spectromicroscopy of single point emitters can be the basis for the methods of "multicolor" (hyperspectral) nanodiagnostics of condensed matter.

This work was supported by Russian Foundation of Basic Researches (17-02-006525 development of the microrefractometry with single molecules); Russian Science Foundation (14-12-01415 - application of statistical methods for SMSM researches).

\section{References}

1. T. Basché, W.E. Moerner, M. Orrit, U.P. Wild, Single-molecule optical detection, imaging and spectroscopy (VCH, Weinheim, Cambridge, 1997)

2. M. Orrit et al., Faraday Discussions 184, 275 (2015)

3. A.V. Naumov, Physics Uspekhi 56, 605 (2013)

4. A.V. Naumov, I.Yu. Eremchev, A.A. Gorshelev, Eur. Phys. J. D 68, 348 (2014)

5. A.M. van Oijen et al., Chem. Phys. Lett. 292, 183 (1998)

6. S.R.P. Pavani et al., Proc. Natl. Acad. Sci. USA 106, 2995 (2009)

7. K. K. Rebane, J. Lumin. 100, 219 (2002)

8. T. Plakhotnik, D. Walser, A. Renn, Phys. Rev. Lett. 77, 5365 (1996)

9. T. Plakhotnik, E.A. Donley, U.P. Wild, Ann. Rev. Phys. Chem. 48, 181 (1997)

10. T. Plakhotnik, J. Lumin. 83-84, 221 (1999)

11. E.A. Donley, T. Plakhotnik, J. Chem. Phys. 114, 9993 (2001)

12. T. Nonn, T. Plakhotnik, Chem. Phys. Lett. 336, 97 (2001)

13. A.V. Naumov et al., Angew. Chemie. Int. Ed. 48, 9747 (2009)

14. A.V. Naumov et al., Phys. Chem. Chem. Phys. 13, 1734 (2011)

15. T.A. Anikushina et al., Faraday Discussions 184, 263 (2015)

16. D.V. Kuznetsov, V.K. Roerich, M.G. Gladush, JETP 113, 647 (2011)

17. D.V. Kuznetsov, V.K. Roerich, M.G. Gladush, Theor. Math. Phys. 168, 1078 (2011)

18. M.Y. Eremchev, I.Y. Eremchev, A.V. Naumov, EPJ Web of Conf. 103, 05003 (2015)

19. M. Nirmal et al., Nature 383, 802 (1996)

20. P. Frantsuzov, M. Kuno, B. Janko, R.A. Marcus, Nature Physics 4, 519 (2008)

21. A.L. Efros, D.J. Nesbitt, Nature Nanotechnology 11, 661 (2016)

22. T. Plakhotnik et al., Phys. Rev. Lett. 105, 167402 (2010)

23. M.J. Fernee et al., J. Phys. Chem. Lett. 3, 1716 (2012)

24. A.L. Shchukina, I.Yu. Eremchev, A.V. Naumov, Phys. Rev. E 92, 032102 (2015)

25. I.S. Osad'ko, I.Yu. Eremchev, A.V. Naumov, J. Phys. Chem. C 119, 22646 (2015)

26. I.Yu. Eremchev, I.S. Osad'ko, A.V. Naumov, J. Phys. Chem. C 120, 22004 (2016)

27. A. Pillonnet et al., Optics Express 20, 3200 (2012) 